\title{
An Aerodynamic Drag Model for Protein Ions
}

\author{
D.J. Douglas \\ Sciex, Thornhill, Ontario, Canada
}

\begin{abstract}
The energy losses of protein ions passing through a collision cell filled with inert gas have been modeled as the aerodynamic drag on a projectile at high Knudsen number. When applied to the energy loss data of Covey and Douglas (J. Am. Soc. Mass Spectrom. 1993, 4, 616-623) with drag coefficients from the gas dynamics literature, derived protein cross sections are $\sim 0.8$ of those found with the simple collision model used by Covey and Douglas. (J Am Soc Mass Spectrom 1994, 5, 17-18)
\end{abstract}

$\mathrm{R}$ ecently, Covey and Douglas [1] have described a method for the determination of collision cross sections of proteir ions. The energy losses of ions passing through a cell filled with gas at $\sim 1 \times$ $10^{-3}$ torr are measured and interpreted with a simple collision model. Similar observations of energy losses have recently been described by Cox et al. [2]. The model used in ref 1 is plausible, but requires several approximations, including (1) assuming an average center-of-mass scattering angle of $90^{\circ}$, (2) neglecting inelastic collisions, (3) modeling the relation between impact parameter and scattering angle by hard sphere collisions, and (4) neglecting the thermal motion of the target gas. The slowing of a projectile passing through a low density gas is a classic problem in gas dynamics which has been extensively studied both theoretically and experimentally. In particular, several groups have measured drag coefficients of small spheres in low density gases [3-10]. The experimental drag coefficients of course include inelastic collisions and scattering through a range of angles from a rough surface. More realistic theories which attempt to incorporate these effects are in fair agreement with the experimental data [11-17]. In the absence of a more detailed understanding of the collision dynamics of protein ions, the concept of aerodynamic drag can be applied to offer an alternate interpretation of the experimental energy loss data of ref 1 . Here it is shown that the experimentally determined drag mefficients give derived protein cross sections similar to those obtained from the simple collision model.

The drag coefficient of a particle passing through a gas, $C_{D}$, can be defined by

$$
m_{1} \frac{d v}{d x}=-\frac{C_{D} A \rho v}{2}
$$

Address reprint requests to D.J. Douglas, Sciex, 55 Glen Cameron Road, Thornhill, Ontario L3T 1P2, Canada. where $m_{1}$ is the particle mass, $v$ is the magnitude of the particle's velocity (speed), $x$ is distance, $A$ is the particle projected area $(\approx$ cross section), and $\rho$ is the gas mass density [7].

For the case where a projectile (e.g., a protein ion) enters a collision cell of length $l$ with speed $v_{0}$ (energy $E_{0}$ ) and exits with speed $\mathrm{v}$ (energy $E$ ), integrating eq 1 and squaring the result gives

$$
E / E_{0}=\left(v / v_{0}\right)^{2}=e\left(\frac{-C_{\alpha} A p l}{m_{1}}\right)
$$

Equation 2 assumes that $C_{D}$ is independent of speed $v$. For the experimental conditions of ref 1 , this is a fair approximation. Thus this model gives an exponential energy loss with increasing gas density as observed in the experiments of ref 1 .

The value of the drag coefficient depends on the ratio of the mean free path of the collision gas, $\lambda_{g^{\prime}}$ to the projectile's diameter, $D$, that is, the Knudsen number $K_{n}=\lambda_{g} / D$, and the ratio of the speed of the projectile to the thermal speed of the collision gas molecules, that is, the speed ratio s. For the case of protein ions with $30 \mathrm{eV}$ to $410 \mathrm{eV}$ energy entering a collision cell with a gas pressure of $\sim 1 \times 10^{-3}$ torr, the Knudsen number is very high $\left(\sim 10^{6}\right)$. (The Reynolds number is nearly zero.) The speed ratio depends on the ion mass and kinetic energy. For all of the ions studied in ref $1, s$ ranges from 2.5 to 5.0 and typically $s \approx 3$. The experiments [3-10] and theories [11-17] cited show that under these conditions, $C_{D}$ $\approx 2.5$. Fitting the exponential energy loss data of ref 1 to eq 2 with $C_{D} \approx 2.5$ gives in every case protein cross sections which are about $80 \%$ of those derived from the simple collision model. For myoglobin +9 ions with $90 \mathrm{eV}$ energy, for example, $s=2.7$, giving $C_{D}$ $\approx 2.4$. The calculated cross section from eq 2 then is $2140 \AA^{2}$. The value calculated from the simple collision model of ref 1 is $2570 \AA^{2}$. The relation of the cross sections calculated from the drag coefficients to those 
calculated in ref 1 can be seen by comparing eq 2 here to eq 12 of ref 1 which fits energy losses to

$$
E / E_{0}=e^{-\boldsymbol{s} S(\ln \alpha)}
$$

where $\sigma$ is the collision cross section, $S$ the target thickness (product of target gas number density $n$ and cell length $l$ ), and $\alpha^{\prime}$ is given by

$$
\alpha^{\prime}=M^{2} /\left(m_{1}^{2}+m_{2}^{2}\right)
$$

where $m_{1}$ is the projectile (ion) mass, $m_{2}$ is the collision gas mass, and $M=m_{1}+m_{2}$. For measured energy ratios $E / E_{0}$, fitting the data to eq 2 will give the area $A$ in the aerodynamic drag model, and fitting the data to eq 3 will give the cross section $\sigma$ in the collision model:

$$
E / E_{0}=e\left(\frac{-C_{d} A \rho l}{m_{1}}\right)=e^{-\sigma S\left(l n \alpha^{\prime}\right)}
$$

Equating the exponentials, rearranging, and noting $\rho / n=m_{2}$, gives the ratio of the cross section determined from the collision model $\sigma$, to that determined from the aerodynamic drag model $A$, as

$$
\sigma / A=\left(C_{D} m_{2}\right) /\left(m_{1} \ln \left(\alpha^{\prime}\right)\right)
$$

Further, where the mass of the protein ion, $m_{1}$, is much greater than $m_{2}$

$$
\alpha^{\prime}=M^{2} /\left(m_{1}^{2}+m_{2}^{2}\right) \approx 1+2 m_{2} / m_{1}
$$

and so $\ln \left(\boldsymbol{\alpha}^{\prime}\right) \approx 2 m_{2} / m_{1}$ and

$$
\sigma / A \approx C_{n} / 2
$$

The model of ref 1 involves specular reflection of hard spheres at infinite speed ratio which, for $m_{1}$, much greater than $m_{2}$, gives $C_{D}=2.0$ (see, e.g., ref 17). Since the experimental value of $C_{D}$ is 2.5 , the ratio $\sigma / A$ is $\sim 1.25$ (or $A / \sigma=0.8$ ). It is encouraging that the cross sections determined by using more realistic values for $C_{D}$ are not too different from those of the simpler model. In both cases, of course, relative cross sections are the same. Both experiment and theory show that the value of $C_{D}$ increases for lower Knudsen numbers and for lower speed ratios. This suggests that determination of protein cross sections by other techniques, such as ion mobility, may yield different values if these effects are not considered.

\section{References}

1. Covey, T. R.; Douglas, D. J. J.Am. Soc. Mass Spectrom, 1993, 4 616-623.

2. Cox, K. A.; Julian, R. K.; Cooks, R. G.; Kaiser Jr., R.E. J. Am. Soc. Mass Spectrom. in press 1993.

3. Masson, D. J.; Morris, D. N.; Bloxsom, D.E. In Rarefied Gas Dymamics; Talbot, L., ed; Academic Press: New York, 1961; pp 643-661.

4. Wegener, P. P.; Ashkenas, H. In Rarefied Gas Dynamics; Talbot, L., ed; Academic Press: New York, 1961; pp 663-667.

5. Ashkenas, H. In Rarefied Cas Dynamics; Laurmann, J. A., ed,; Academic Press: New York, 1963; pp 278-290.

6. Aroesty, J. A. In Rarefied Gas Dynamics; Laurmann, J. A., ed, Academic Press: New York, 1963; pp 261-277.

7. Hersh, A.S.; Friichtenacht, J.F.; Slattery, J. C. In Rarefied Gas Dynumics; Trilling, L.; Wachman, H. Y., eds,; Academic Press: New York, 1969, pp 757-766.

8. Sims, W. H. In Rarefied Cas Dynamics; Trilling, L.; Wachman, H. Y., eds.; Academic Press: New York, 1969; pp 751-756.

9. Potter, J. L.; Miller, J. T. In Rarefied Gas Dynamics; Trilling, L.; Wachman, H. Y., eds.; Academic Press: New York, 1969; pp 723-743.

10. Phillips, W. M; Kulthau A. R. In Rarefied Cas Dynamics; Trilling, L.; Wachman, H. Y., eds.; Academic Press: New York, 1969; pp 711-720.

11. Epstcin, P. S. Phys. Reo. 1923, 23, 710-723.

12. Stalder, J. R.; Zurich, V. J. National Advisory Committee for Aeronautics; Technical Note 2423, 1951.

13. Robertson S. J. In Rarefied Gas Dynamics; Trilling, L.; Wachman, H. Y., eds.; Academic Press: New York, 1969; Pp $767-777$.

14. Henderson, C. B. ALAA Joumal, 1976, 14, 707-708.

15. Larina, I. N.; Rykov, V. A. In Rarefied Gas Dynamics; Fisher, S. S., ed.; AIAA: New York, 1980; pp 1023-1030.

16. Gspann, J.; Vollmar, H. In Rarefied Gas Dynamics; Campargue, R., ed.; CEA: Paris, 1979; pp 1193-1206.

17. Gspann, J. In Rarefied Gas Dyramics; Fisher, S. S., ed.; AIAA: New York, 1980; pp 959-963. 